\title{
Corrigendum: Carbon precipitation from heavy hydrocarbon fluid in deep planetary interiors
}

Sergey S. Lobanov, Pei-Nan Chen, Xiao-Jia Chen, Chang-Sheng Zha, Konstantin D. Litasov, Ho-Kwang Mao \& Alexander F. Goncharov

Nature Communications 4:2466 doi:10.1038/ncomms3446 (2013); Published 12 Sep 2013; Updated 26 Nov 2013

The financial support for this article was not fully acknowledged. The Acknowledgements should have included the following:

Use of the Advanced Photon Source was supported by the US Department of Energy, Office of Science, Office of Basic Energy Sciences, under contract number DE-AC02-06CH11357. GSECARS at APS is supported by the National Science Foundation Earth Sciences (EAR-0622171) and Department of Energy Geosciences (DE-FG02-94ER14466). 